\title{
Effect of Advanced Glycation End Products on Lectin-Like Oxidized Low Density Lipoprotein Receptor-1 Expression in Endothelial Cells
}

\author{
Sammy WM Shiu, Ying Wong and Kathryn CB Tan
}

Department of Medicine, University of Hong Kong, Hong Kong

\begin{abstract}
Aim: Lectin-like oxidized LDL receptor-1 (LOX-1) is a class E oxidized LDL specific scavenger receptor that recognizes multiple ligands. Advanced glycation end products (AGEs) have been recently identified as other ligands to LOX-1 and shown to increase LOX-1 expressions in diabetes; therefore, we investigated the underlying mechanism involved.

Methods: Confluent human aortic endothelial cells were treated with a fixed concentration of AGEBSA or BSA as a control in the presence or absence of either antibody of the receptor for advanced glycation end products, mammalian target of rapamycin (mTOR) inhibitor rapamycin, NF-kB inhibitor, phosphoinositide 3-kinases (PI3K) inhibitor or anti-diabetic drug metformin. After stimulation, cells were lysed and Western blot protein expression on LOX-1, rapamycin-insensitive companion of mTOR (RICTOR), the phosphorylation status of p-mTOR, p-P70S6 kinase and p-Akt were determined.

Results: AGEs induced LOX-1 expression in endothelial cells. Pretreatment either with anti-RAGE antibody or LY294002 prior to AGE-BSA decreases LOX-1 and p-mTOR expressions. Incubating endothelial cells with AGE-BSA in the presence of rapamycin down-regulated the protein expressionlevel of p-mTOR by $41 \%(p<0.05)$ and LOX-1 expression by $61.5 \%(p<0.01)$. Knockdown of RICTOR by RNA silencing showed a $41.5 \%(p<0.01)$ and $71.2 \%(p<0.01)$ reduction in LOX-1 and p-Akt expressions, respectively. Preincubation of endothelial cells with AGE-BSA and metformin, an anti-diabetic drug known to have an mTOR inhibition effect, significantly reduced AGEstimulated LOX-1 expression.

Conclusion: Our results indicated that LOX-1 up-regulation induced by AGE-BSA was a receptor mediated through RAGE and is via the PI3K/PDK1/mTORC2 pathway. Metformincan reduce AGE-stimulated LOX-1 expression in endothelial cells in vitro.
\end{abstract}

J Atheroscler Thromb, 2012; 19:1083-1092.

Key words; Advanced glycation end products (AGEs), Lectin-like oxidized low density lipoprotein receptor-1 (LOX-1), Mammalian target of rapamycin (mTOR)

\section{Introduction}

LOX-1 is a recently cloned class E oxidized LDL specific scavenger receptor that is predominantly expressed in endothelial cells, macrophages and vascular smooth muscle cells ${ }^{1)}$. LOX-1 is a type II glycolmembrane protein spanning $50 \mathrm{kDa}$ in size and con-

Address for correspondence: Kathryn Tan, Department of Medicine, University of Hong Kong, Queen Mary Hospital, Pokfulam Road, Hong Kong

E-mail: kcbtan@hkucc.hku.hk

Received: September 5, 2011

Accepted for publication: June 8, 2012 sists of a short $\mathrm{N}$-terminal, a neck and a long C-terminal extracellular domain ${ }^{2)}$. LOX-1 is a multi-ligand receptor and has been implicated in vascular inflammation, atherosclerotic plague formation and destablization $^{3,4)}$. It can bind oxidized LDL (oxLDL), phospholipids, apoptotic bodies, aged cells, activated platelets, advanced glycation end products (AGEs) and both Gram-positive and Gram-negative bacteria. Among these known ligands, the cellular consequences of oxLDL binding to LOX-1 are the most extensively studied, and activation of LOX-1 by oxLDL has a proatherogenic effect. Ligation of oxLDL to LOX-1 in endothelial cells triggers a number of signaling path- 
ways including p38 (MAPK), p44/42 MAPK, protein kinase $\mathrm{C}(\mathrm{PKC})$ and transcription factor $\mathrm{NF}-\mathrm{kB}^{5-8)}$. This results in increased expression of adhesion molecules and monocyte chemoattractant protein-1 ${ }^{9)}$, release of matrix metalloproteinases, and reduction of endothelial nitric oxide synthase expression ${ }^{10,11)}$. In vascular smooth muscle cells, ligation of oxLDL to LOX-1 has been reported to induce apoptosis ${ }^{12)}$ and in macrophages, uptake of oxLDL by LOX-1 leads to foam cell formation ${ }^{13)}$.

LOX-1 is not constitutively expressed and the expression of LOX-1 can be induced by a number of stimuli including oxLDL ${ }^{14,15)}$, shear stress ${ }^{16)}$, inflammatory cytokines ${ }^{17-19)}$ and angiotensin $\Pi^{20)}$. We and other groups have shown that AGEs can also induce LOX-1 expression ${ }^{17,21)}$. Since there is increased formation and accumulation of AGEs in diabetes mellitus, AGEs may contribute to the increased expression in LOX-1 seen in human diabetes ${ }^{17,22)}$. In contrast to oxLDL, which has been shown to induce LOX-1 expression via activation of $\mathrm{NF}-\mathrm{kB}^{14}$ ), the intracellular signaling pathways involved in AGEs-stimulated LOX-1 expression have not been delineated. In the present study, we have shown that, unlike oxLDL, pretreatment with NF-kB inhibitor does not reduce AGEs-induced LOX-1 expression. This would suggest that stimulation of LOX-1 expression by AGEs is not mediated via activation of NF-kB. On the other hand, rapamycin, an inhibitor of the mammalian target of rapamycin (mTOR), can markedly reduce AGEsstimulated LOX-1 expression in endothelial cells in vitro. We have further elucidated the role of mTOR signaling in the regulation of LOX-1 expression in AGE-stimulated endothelial cells, and examined the effect of metformin, an anti-diabetic drug that has an mTOR inhibition effect ${ }^{23,24)}$, on AGE-stimulated LOX-1 expression.

\section{Methods}

\section{AGE-BSA Preparation}

AGE-BSA was prepared by incubating $20 \mathrm{mg} /$ mL BSA (fraction V, low endotoxin; Invitrogen) with $0.5 \mathrm{M}$ glucose in $\mathrm{PBS}, \mathrm{pH} 7.2$, at $37^{\circ} \mathrm{C}$ under sterile conditions. After 90 days of incubation, unbound lowmolecular-weight products were removed by extensive dialysis against PBS. The degree of glycation on BSA (AGE-BSA fluorescence) was measured by spectrofluorometric detection at excitation of $370 \mathrm{~nm}$ and emission of $440 \mathrm{~nm}^{25)}$.

\section{Low-Density Lipoprotein Isolation and Modification Low-density lipoprotein $(\mathrm{d}=1.02-1.05 \mathrm{~g} / \mathrm{mL})$}

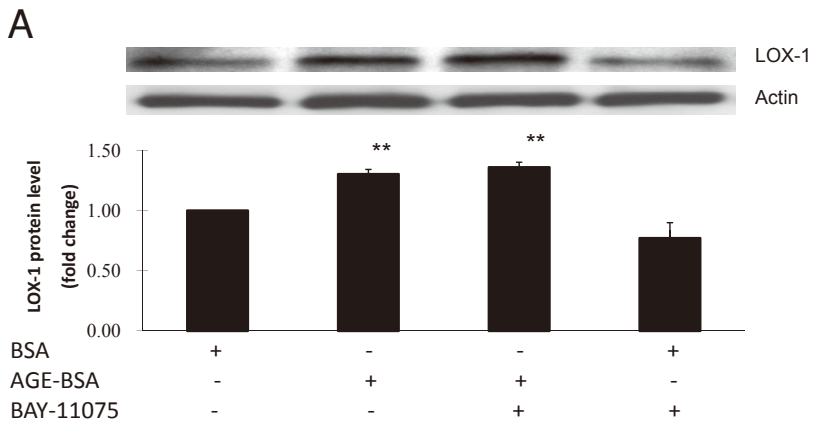

B
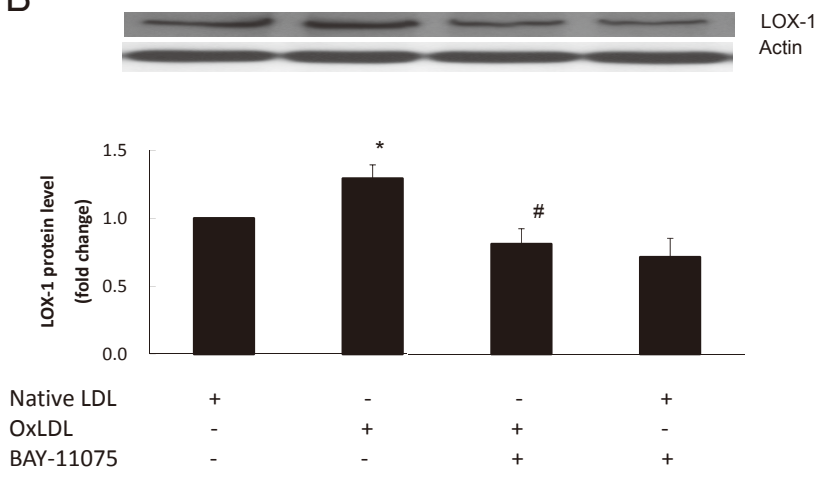

Fig. 1. Effect of NF-Kappa B inhibition on LOX-1 expression in AGE-BSA-treated endothelial cells (1A) and oxLDL-treated endothelial cells (1B). HAECs were pre-treated with the NF-Kappa B inhibitor (BAY11075, $40 \mathrm{uM)} 1$ hour before incubation with either $100 \mathrm{ug} / \mathrm{mL}$ AGE-BSA or $100 \mathrm{ug} / \mathrm{mL}$ oxLDL. Inhibition of NF-Kappa B blocked oxLDL-induced LOX-1 expression but had no effect on AGE-BSA-induced LOX-1 expression. ${ }^{* *} p<0.01$ versus BSA-treated cells and ${ }^{*} p<0.05$ versus native LDL-treated cells, ${ }^{\#} p<$ 0.05 versus oxLDL-treated cells.

was prepared by sequential ultracentrifugation ${ }^{26)}$. Oxidized LDL was prepared by incubating freshly prepared LDL $(1 \mathrm{mg} / \mathrm{mL})$ with $5 \mu \mathrm{M} \mathrm{CuCl}_{2}$ in $0.1 \mathrm{~mol} /$ $\mathrm{L}$ phosphate-buffered saline (PBS) in the dark for 16 $h$ at $37^{\circ} \mathrm{C}$. Native LDL was used as a control and was prepared by incubating LDL with $1 \mathrm{mM}$ EDTA and 1 mM DTPA for 16 hours. The modification was stopped by extensive dialysis of the reaction mixture against $0.15 \mathrm{M} \mathrm{NaCl}$ and $1 \mathrm{mM}$ EDTA at $4^{\circ} \mathrm{C}^{27}$.

\section{Cell Culture}

Human aortic endothelial cells (HAEC) (Clonetics, San Diego, CA) were grown to confluence in EGM-1 Bullet Kit Medium and passages 5-8 were used throughout the experiments. Confluent human aortic endothelial cells were kept overnight in serumfree, supplement-free EGM-1 medium and subse- 
A

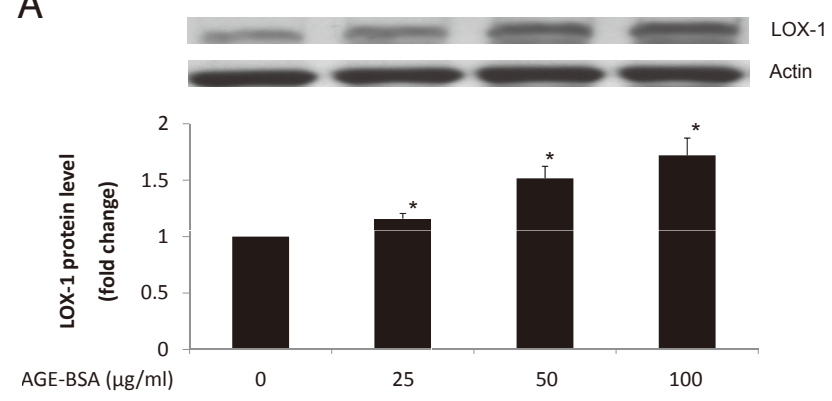

B

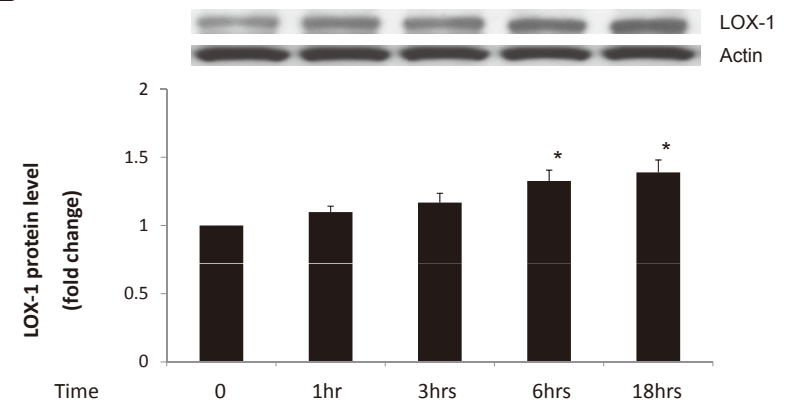

Fig. 2. Effect of AGE-BSA on LOX-1 expression in endothelial cells. Cells were incubated with increasing doses of AGE-BSA for 24 hours (2A) or increasing incubation time at a fixed concentration of $100 \mathrm{ug} / \mathrm{mL}$ AGE-BSA (2B). ${ }^{*} p<0.05$ versus BSA-treated cells.

quently treated with a fixed concentration of $100 \mu \mathrm{g} /$ mL AGE-BSA in the presence or absence of mTOR inhibitor rapamycin, phosphoinositide 3-kinases (PI3K) inhibitor (LY294002), NF-kB inhibitor (BAY11-7085), metformin (Calbiochem, Rockland, MA, USA), antibody of receptor for advanced glycation endproducts, anti-RAGE (R\&D, MN) or siRNA (on RICTOR or RAPTOR; Ambion, Grand Island, NY, USA).

\section{Transfection of siRNA}

RICTOR, regulatory associated protein of mTOR (RAPTOR) and scrambled control siRNA were purchased from Ambion. HAEC cells were transfected with $100 \mathrm{nM}$ siRNA in Optimem medium (Invitrogen, Grand Island, CA, USA) mixed with Lipofectamine 2000 according to the manufacturer's instructions. After six hours of transfection, cells were starved for 12 hours and finally HAEC cells were treated with the indicated AGE-BSA concentrations for another six hours.
A

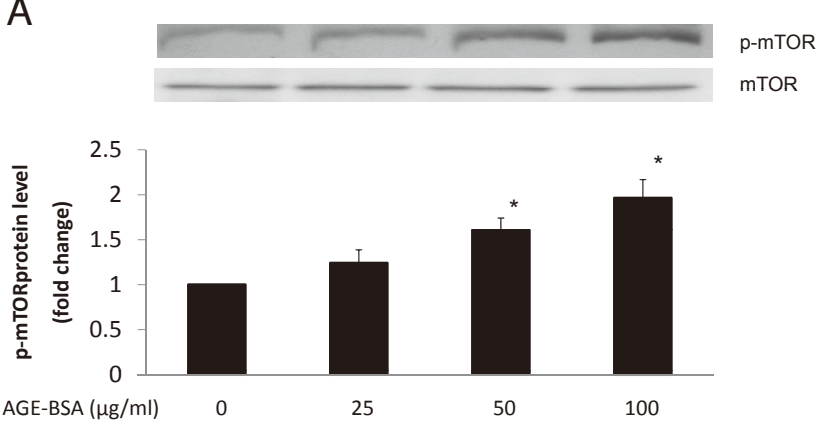

B

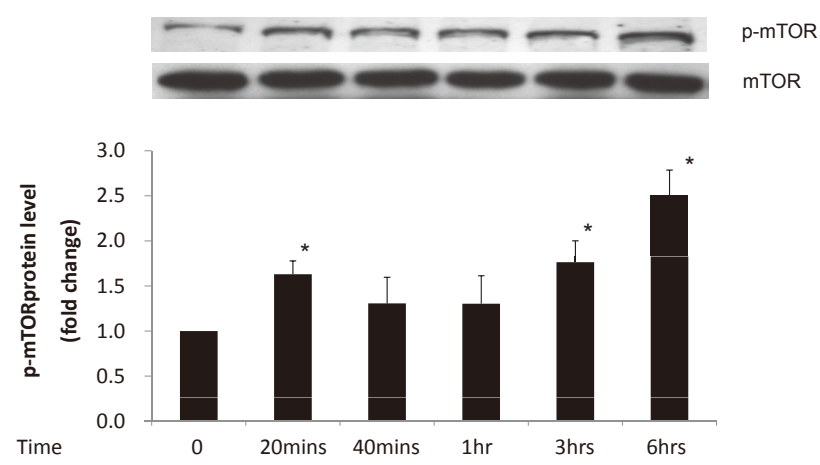

Fig. 3. Effect of AGE-BSA on phosphorylation of mTOR in endothelial cells. Cells were incubated with increasing doses of AGE-BSA for 24 hours (3A) or increasing incubation time at a fixed concentration of $100 \mathrm{ug} /$ $\mathrm{mL}$ AGE-BSA (3B). ${ }^{*} p<0.05$ versus BSA-treated cells.

\section{Western Blot Analysis}

After stimulation, HAEC cells were washed and scraped in ice-cold PBS. After lysis in RIPA buffer, cells were further sonicated and cell supernatants were quantified by the Lowry protein assay (Biorad, Hercules, CA, USA). Then, 25 ug protein of total cell lysates was electrophoresed on $7.5 \%$ SDS-polyacrylamide gel followed by immunoblotting onto a polyvinylidene difluoride membrane (PVDF). The membrane was subsequently probed against primary antibodies specific to LOX-1 (1:1000; R\&D), RICTOR, RAPTOR, and mTOR (1:1000; Cell Signaling, MA). To determine the phosphorylation status of AGE-treated endothelial cells, phospho-specific antibodies (1:1000; Cell Signaling) of p-mTOR(Ser2448), p-P70S6 (Thr389) (substrates of mTORC1 complex) and p-Akt (Ser473) (substrates of mTORC2 complex) underwent Western blot analysis as shown above. Secondary antibody of anti-rabbit IgG (1:2500) conjugated with horseradish peroxidase (HRP) was then applied to the membrane before X-ray film autoradiography. Betaactin was employed for standardization. All experi- 
A
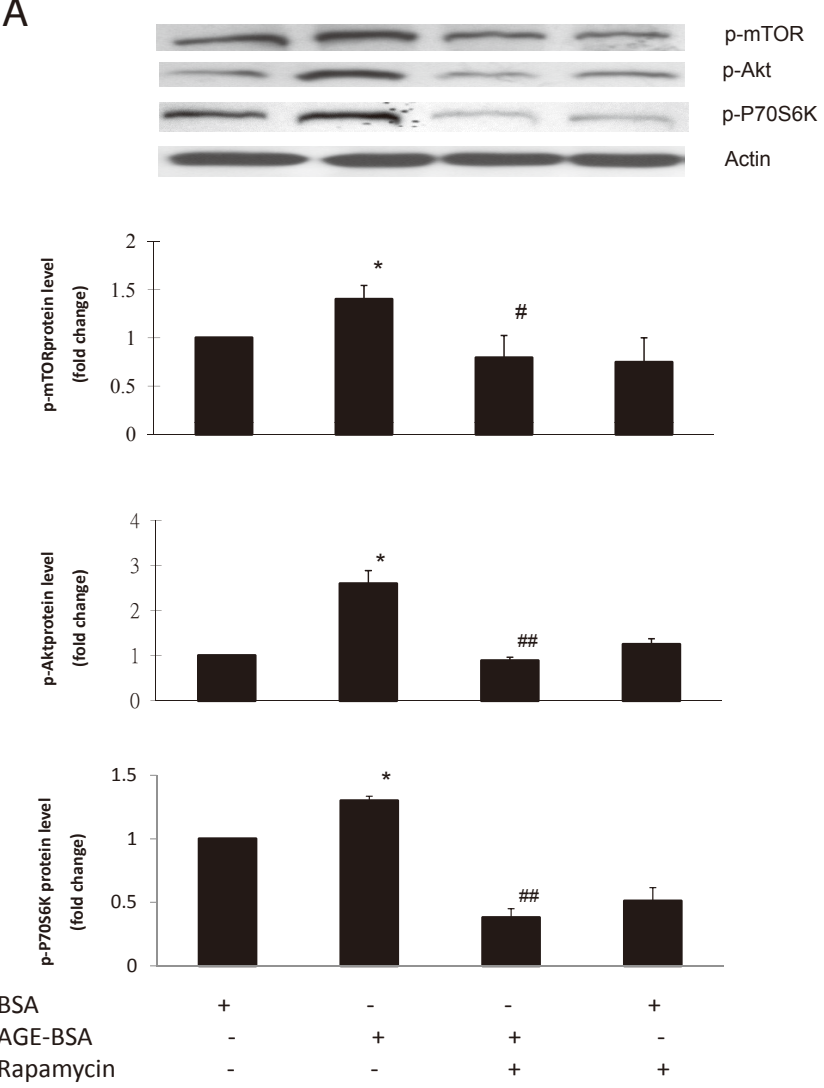

B

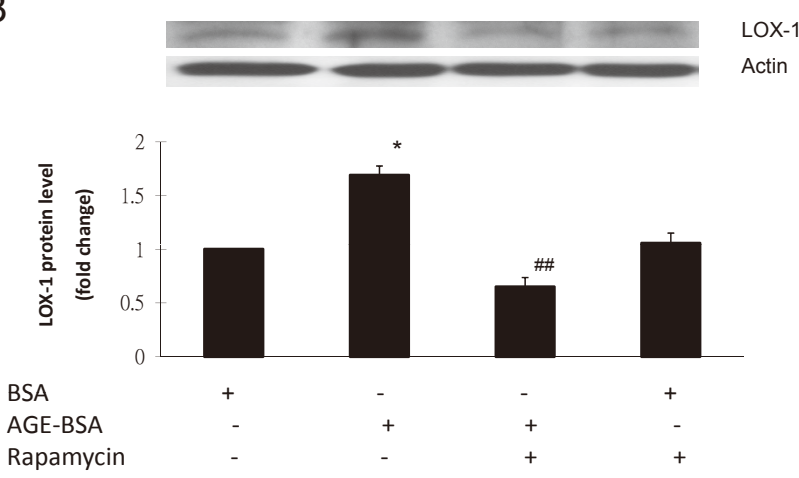

C

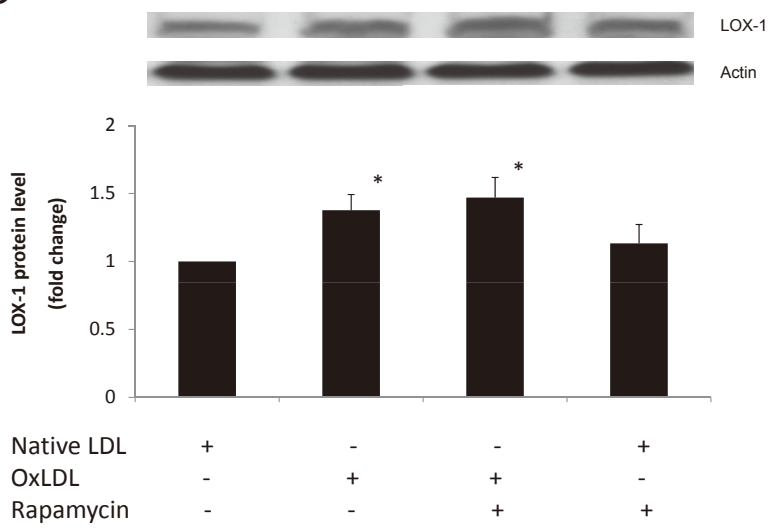

Fig. 4. AGE-BSA increased LOX-1 expression in HAECs via activation of mTOR pathway. HAECs were incubated with $100 \mathrm{ug} /$ $\mathrm{mL}$ AGE-BSA for 6 hours and Western blot analysis showed increased phosphorylation of p-mTOR, p-Akt and p-P70S6K $(4 \mathrm{~A})$ and LOX-1 expression (4B). These effects were abolished by the addition of the mTOR inhibitor rapamycin $(1 \mathrm{uM})$. ${ }^{*} p<0.05$ versus BSA-treated cells, and ${ }^{\#} p<0.05,{ }^{\# \#} p<0.01$ vs AGE-BSA-treated cells. Rapamycin blocked AGE-BSA induced LOX-1 expression but had no effect on oxLDL-induced LOX-1 expression (4C). ${ }^{*} p<0.05$ versus native LDLtreated cells.

ments were repeated 3 to 5 times and the results are expressed asthe mean \pm SD.

\section{Results}

Both AGE-BSA and oxLDL induced LOX-1 expression in HAEC cells but, unlike oxLDL, the effect of AGE-BSA on LOX-1 expression was not blocked by NF-kB inhibitor (Fig. 1). Adding AGEBSA to endothelial cells led to a dose- and timedependent increase in LOX-1 expression (Fig. 2). To investigate whether the upregulation of LOX-1 by AGE-BSA was mediated by the mTOR pathway, AGE-BSA was added to endothelial cell culture and phosphorylation of p-mTOR was measured. Our data showed that AGE-BSA stimulated the phosphorylation of p-mTOR in a dose- and time-dependent manner (Fig. 3). Since mTOR forms two distinct functional complexes, termed mTORC1 and mTORC2, downstream targets of both mTORC1 and mTORC2 were also evaluated. AGE-BSA increased the phosphorylation of p-mTOR (1.4-fold $\pm 0.1, p<0.05)$, and predominantly the downstream target of mTORC2, p-Akt (2.6-fold $\pm 0.3, p<0.05)$ and had a lesser effect on the downstream target of mTORC1, p-P70S6 kinase (1.3-fold $\pm 0.1, p<0.05)$ (Fig. 4A). Rapamycin, a specific mTOR inhibitor, was used in our experiments to inhibit the mTOR pathway. As expected, incubating endothelial cells with AGE-BSA in the presence of rapamycin down-regulated the level of protein expressions of p-mTOR, p-Akt and p-P70S6K (Fig. 4A). This resulted in a reduction of LOX-1 expression by $61.5 \%$ compared to AGE-BSAtreated cells as a control $(p<0.01)$ (Fig. 4B). Taken together, these data would suggest that AGE-BSA increased LOX-1 in HAEC cells via phosphorylation of the mTOR pathway. Unlike AGE-BSA, incubating endothelial cells with oxLDL in the presence of rapa- 
A

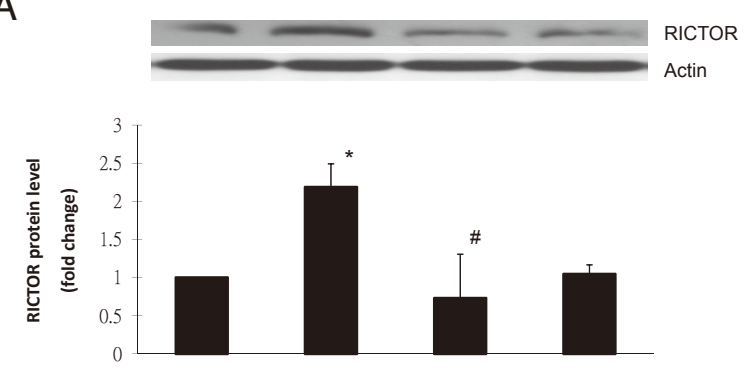

B
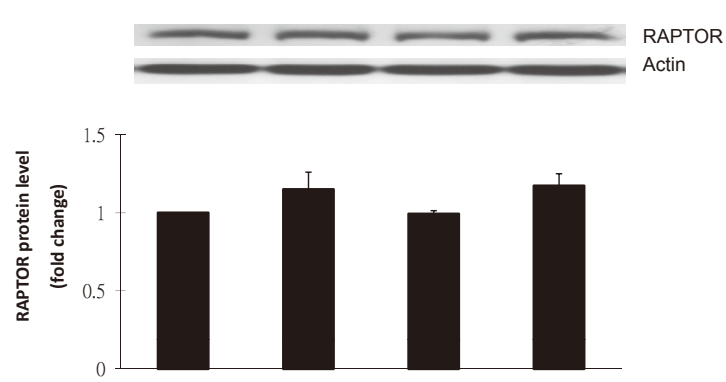

BSA

AGE-BSA

Rapamycin

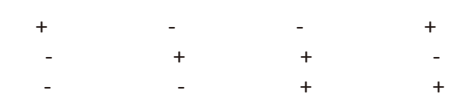

$\mathrm{C}$

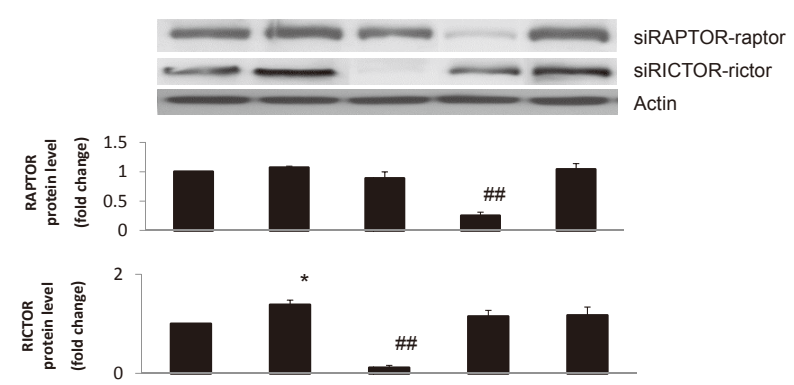

$\mathrm{D}$

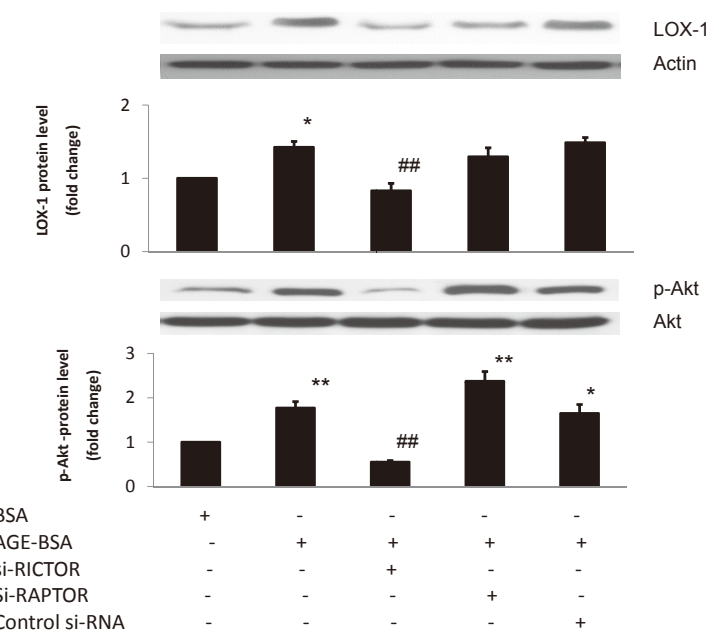

Fig. 5. AGE-BSA induced LOX-1 expression in HACEs mainly via mTORC2. HACEs were incubated with $100 \mathrm{ug} / \mathrm{mL}$ AGE-BSA for 6 hours and Western blot analysis showed an increase in RICTOR expression (5A) but no similar increase in RAPTOR expression (5B). The increase in RICTOR expression was abolished by the addition of rapamycin $(1 \mathrm{uM}) .{ }^{*} p<0.05$ versus BSA control and ${ }^{*} p<0.05$ vs AGE-BSA-treated cells.

5C) HAECs were transfected with RAPTOR, RICTOR or CONTROL siRNA. Western blot analysis confirmedtheefficiency of knockdown 48 hours after transfection. ${ }^{\#} p=0.01$ versus AGE-BSA-treated cells.

5D and 5E) Transfected HAECs were incubated with 100ug/ml AGE-BSA for 6 hours. Knockdown of RICTOR, but not RAPTOR, reduced LOX-1 and p-Akt expressions $\left({ }^{*} p<0.05,{ }^{* *} p<0.01\right.$ versus BSA-treated cells, ${ }^{\#} p<0.01$ vs AGE-BSA-treated cells).

mycin showed no reduction of LOX-1 expression, indicating that the mTOR pathway is not involved in the activation of LOX-1 by oxLDL in endothelial cells (Fig. 4C).

To further delineate whether AGE-BSA upregulates LOX-1 mainly via mTORC1 and/or mTORC2, we measured the expression of RAPTOR and RICTOR, the two associated binding proteins of mTORC1 and mTORC2, respectively. Incubation of endothelial cells with AGE-BSA had no significant effect on RAPTOR expression whereas RICTOR expression was significantly increased by 2.2 -fold \pm $0.3, p<0.05$. Treatment of rapamycin prior to AGEBSA stimulation of HAEC showed a marked reduction of RICTOR protein expression by $66.7 \%(p<$ 0.05) (Fig. 5A), but had no significant effect on RAPTOR protein expression (Fig. 5B). Experiments were also performed using RAPTOR or RICTOR siRNA to inhibit mTORC1 or mTORC2, respectively. RICTOR and RAPTOR siRNA specifically blocked RICTOR and RAPTOR expression while control siRNA had no effect (Fig. 5C). Western blotting showed that RICTOR siRNA significantly decreased both LOX-1 expressions by $41.5 \%(p<0.01)$ (Fig. 5D) and p-Akt by $71.2 \%(p<0.01)$ (Fig. 5E), whereas no changes were observed with RAPTOR siRNA.

To investigate whether upstream activation of the mTOR pathway by AGE-BSA is receptor-mediated by the receptor for advanced glycation end products (RAGE), HAEC cells were pretreated with anti-RAGE antibody (R\&D, MN) 1 hour prior to AGE-BSA incubation. Blocking RAGE activation with AGE-BSA led to a $58.4 \%$ reduction $(p<0.05)$ in the level of phosphorylation of p-mTOR (Fig. 6A) and a 33.3\% 


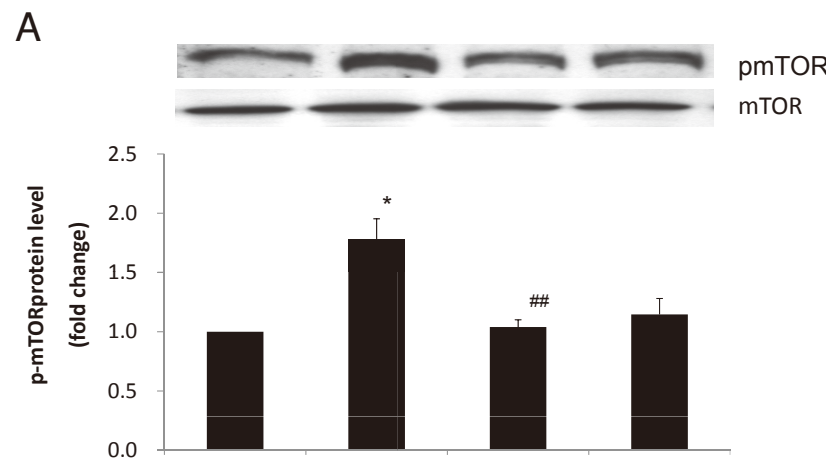

B

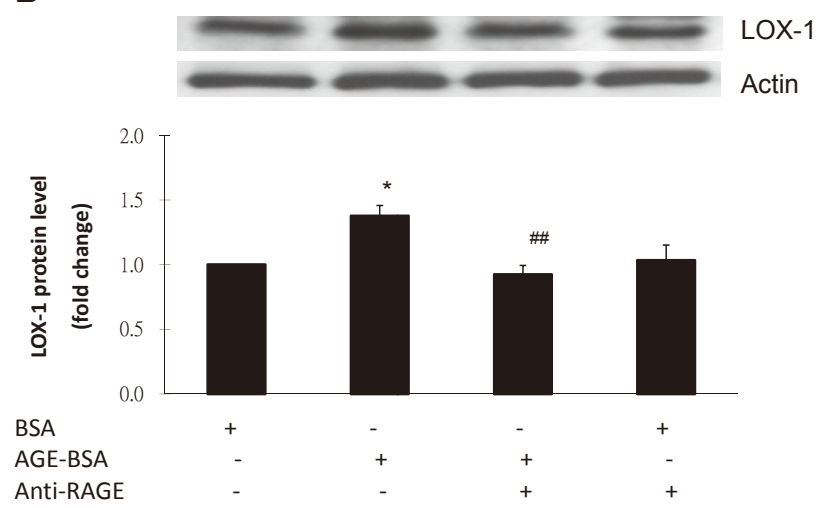

Fig. 6. Inducible LOX-1 expression by AGE-BSA is receptormediated through receptor for advanced glycation end products (RAGE). HAECs were pre-treated with 20 $\mathrm{ug} / \mathrm{mL}$ anti-RAGE antibody for 1 hour before the addition of $100 \mathrm{ug} / \mathrm{mL}$ AGE-BSA for a further 6 hours. Anti-RAGE antibody blocked AGE-BSAinduced phosphorylation of mTOR (6A) and LOX-1 expression $(6 \mathrm{~B}) .{ }^{*} p<0.05$ versus BSA-treated cells, and ${ }^{\# \#} p<0.01$ vs AGE-BSA-treated cells.

$(p<0.01)$ reduction in LOX-1 expression compared to AGE-BSA-treated cells asacontrol (Fig. 6B).

Since ligation of RAGE is known to activate $\mathrm{PI} 3 \mathrm{~K}^{28)}$, experiments were performed to determine whether RAGE-mediated mTOR activation is via PI3K. HAEC cells were treated with LY294002 (a PI3K inhibitor) 1 hour before AGE-BSA stimulation. This resulted in a decrease in the expression level of p-PDK1, a downstream effector molecule of the PI3K pathway, by $36.3 \%(p<0.05)$ (Fig. 7A), and a reduction in the phosphorylation level of p-mTOR by 56.8\% $(p<0.01)$ (Fig. 7B). This was accompanied by a $43.4 \%(p<0.01)$ reduction in LOX-1 expression compared to AGE-BSA-treated cells as a control (Fig. 7C).

Our data have so far shown that AGEs induced LOX-1 expression in endothelial cells via binding to
A

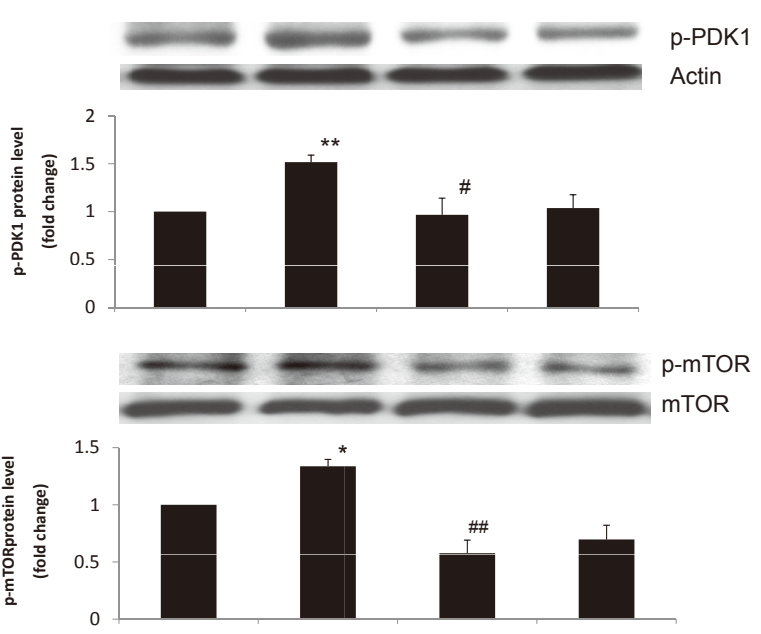

C

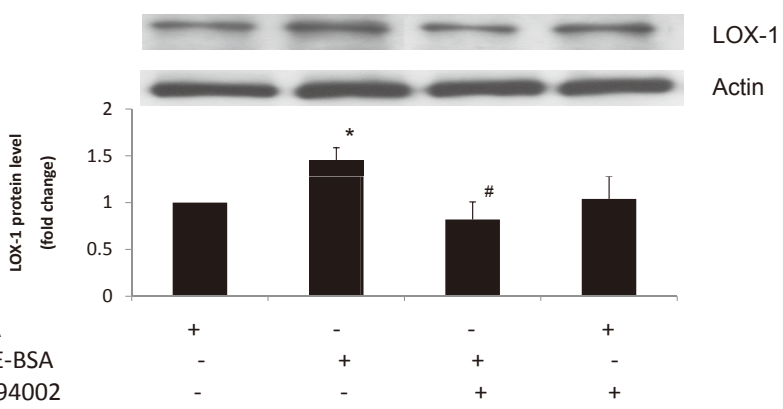

Fig.7. AGE-BSA-stimulated LOX-1 expression required the activation of PI3K. HAECs were pretreated with PI3K inhibitor LY294002 (20 uM) before incubation with $100 \mathrm{ug} / \mathrm{mL}$ AGE-BSA for 6 hours. Inhibition of PI3K led to a reduction of p-PDK1, a downstream effector of PI3K (7A), p-mTOR (7B), and LOX-1 expression (7C). ${ }^{*} p<0.05,{ }^{* *} p<0.01$ versus BSA-treated cells, and ${ }^{\#} p<0.05,{ }^{\# \#} p<0.01$ vs AGE-BSA-treated cells.

RAGE and activation of the mTOR pathway. Since metformin is an anti-diabetic drug known to have an inhibitory effect on mTOR signaling, we therefore investigated whether metformin can attenuate the effect of AGEs on LOX-1 expression in endothelial cells. Our data showed that AGE-BSA increased RAGE expression by 1.4 -fold $(p<0.05)$ and adding metformin did not change RAGE expression in AGEstimulated endothelial cells; however, the addition of metformin reduced p-mTOR by $41.4 \% \quad(p<0.01)$ and decreased LOX-1 expression by $61.5 \%(p<0.01)$ in these cells (Fig. 8).

\section{Discussion}

Diabetes is associated with a high risk of atherosclerosis and recent evidence suggests that the scaven- 
A
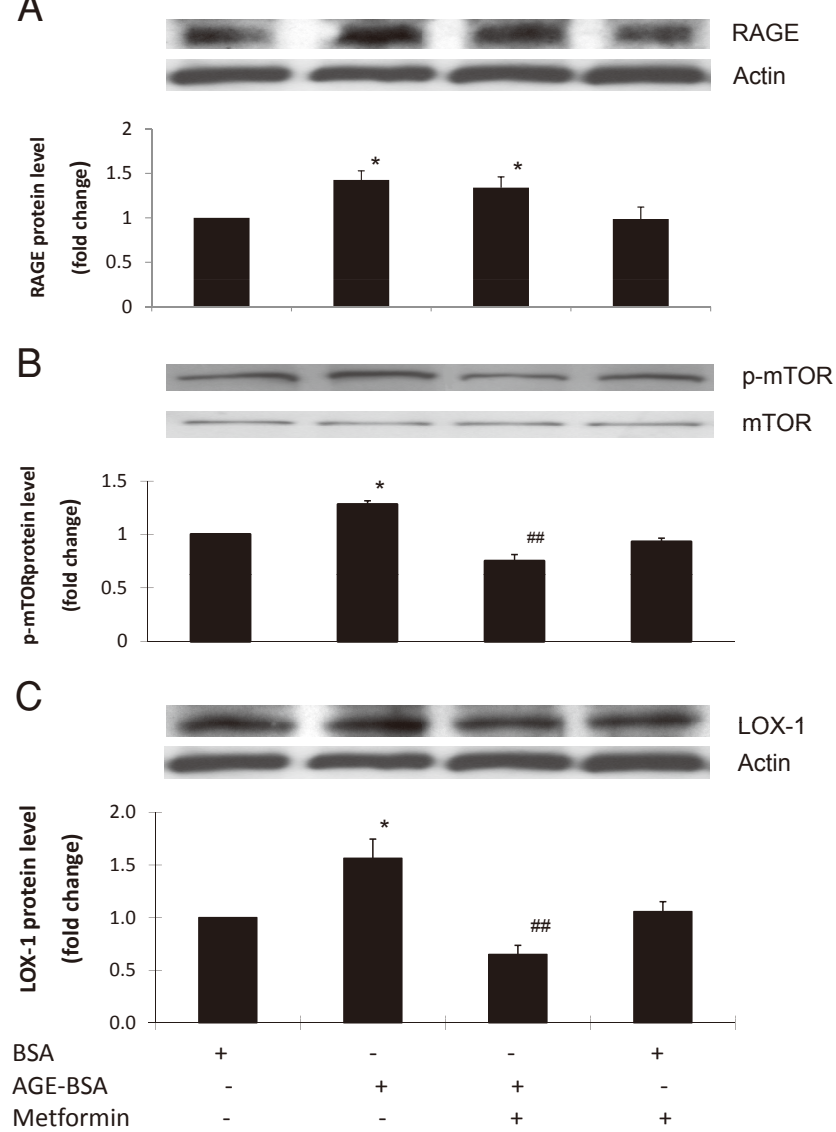

Fig. 8. Effect of metformin on AGE-BSA-stimulated LOX-1 expression in HAECs. HAECs were pretreated with metformin $(2 \mathrm{mM})$ for 1 hour before incubation with AGE-BSA (100 ug/mL) for 6 hours. Metformin had no effect on RAGE expression in AGE-BSA-treated cells (8A) but inhibited AGE-BSA-induced p-mTOR (8B) and LOX-1 expression (8C). ${ }^{*} p<0.05$ versus BSA control and ${ }^{\# \#} p<0.01$ vs AGE-BSA-treated cells.

ger receptor LOX-1 may be involved in the process of atherogenesis in diabetes. Immunohistochemical analysis of diabetic rat aorta sections and human atherosclerotic plaques showed distinct elevated LOX-1 staining and provided evidence to suggest that LOX-1 expression is increased in the vasculature ${ }^{29,30)}$. We have previously demonstrated that both LOX-1 and soluble LOX-1 expression in endothelial cell culture can be upregulated by AGEs in vitro ${ }^{21)}$. Our current data suggest that mTOR is a critical signaling molecule involved in the upregulation of LOX-1 by AGEs, as the increase in LOX-1 is abolished by use of the mTOR inhibitor rapamycin. mTOR is a conserved serine/threonine kinase that regulates cell cycle progression, protein translation, metabolism, and cellular proliferation. There is growing evidence indicating

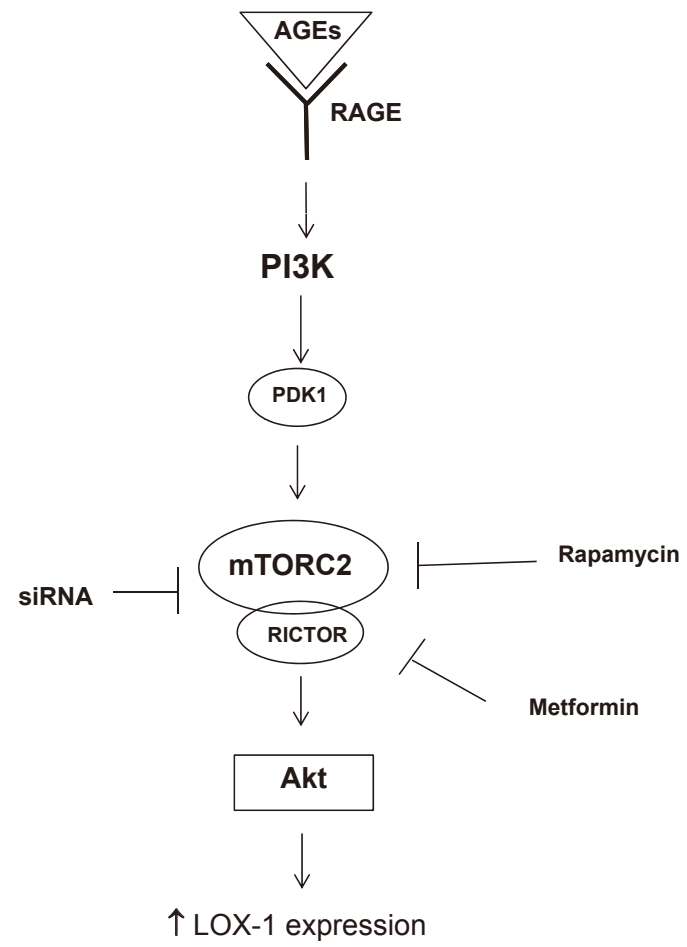

Fig. 9. Proposed signaling pathway involved in AGE-induced LOX-1 expression in endothelial cells. AGEs bind to their cellular receptor, RAGE, and activate $\mathrm{PI} 3 \mathrm{~K} /$ PDK1 with subsequent phosphorylation of mTOR. Triggering of mTOR pathway is mainly via mTORC2/ Akt signaling pathway and thereby induces LOX-1 expression.

that mTOR has a role in cardiac hypertrophy ${ }^{31)}$ and cardiovascular-related disorders such as atherosclero$\operatorname{sis}^{32)}$, and our data add to the body of evidence implicating $\mathrm{mTOR}$ in atherosclerosis.

mTOR exists in two different protein complexes, mTORC1 and mTORC2, which have distinct physiological functions and are regulated by different mechanisms. mTORC1 is composed of mTOR, RAPTOR, mLST8 and PRAS40 and regulates a number of major cellular processes including nutrient metabolism, ribosome biogenesis, and mRNA translation. mTORC2 consists of mTOR, mLST8, sin1, RICTOR, Protor and PRR5 and is found to regulate Akt activity and cytoskeleton arrangement ${ }^{33-35)}$. Rapamycin is an mTOR inhibitor and recent data suggest that the inhibition of mTORC1 and mTORC2 by rapamycin may be dose- and time-dependent. Rapamycin interacts with the intracellular receptor FK506 binding protein 12 (FKBP12), forming a complex with high affinity for mTOR, and preferentially disrupts mTORC1 activity. At higher concentrations of rapamycin, there is FKBP12-independent suppression of 
mTOR signaling. Prolonged rapamycin treatment inhibits the assembly of mTORC2 and reduces the levels of mTORC2 to below those needed to maintain $\mathrm{Akt} / \mathrm{PKB}$ signaling. Rapamycin used in our experiments was in the medium range concentration ( $1 \mathrm{uM})$ and we used a shorter incubation time than recently published studies ${ }^{36,37)}$. This concentration of rapamycin applied to AGE-BSA-stimulated EC culture was able to suppress both $\mathrm{mTORC} 1$ and $\mathrm{mTORC} 2$ activities. Using the RNA silencing technique, knockdown of RICTOR but not RAPTOR led to a marked decrease of the phosphorylation status of Akt ( $\mathrm{p}-\mathrm{Akt}$ ) and LOX-1 expression upon AGE incubation. Hence, mTORC2, but not mTORC1, is the major associated protein complex responsible for the upregulation of LOX-1 expression under AGE stimulation. Taken together, mTORC2 and Akt are the key regulatory signaling molecules for AGE-induced expression of LOX-1. Mukai et al. have also shown that the induction of LOX-1 expression by heparin-binding epidermal growth factor-like growth factor in vascular smooth muscle cells is mediated partly via Akt signaling $^{38)}$.

We also investigated the upstream activation of the mTOR by AGEs and showed that the induction of LOX-1 expression by AGEs is first mediated by its specific receptor RAGE, and the subsequent activation of $\mathrm{mTOR}$ is through the upstream signaling cascade PI3K/PDK1. This is similar to the findings of Dormond et al. who reported that the expression of vascular endothelial growth factor induced by CD40 was mediated by the mTOR pathway in endothelial cells via theupstream activation of $\mathrm{PI} 3 \mathrm{~K}^{39)}$. RAGE is a multi-ligand receptor and ligation of RAGE by its ligands has been shown to activate varios signal transduction cascades, including the family of mitogenactivated protein kinases (MAPKs), members of the JAK-STAT signaling family, CDC42, RAC1 and other members of the Ras family, SRC1, members of the SMAD signaling family and PI3K ${ }^{28)}$. The activation of RAGE by AGEs has been shown to cause endothelial dysfunction with increased expression of vascular cells. In addition to these proatherogenic effects we have shown for the first time that AGE/ RAGE interaction can induce LOX-1 expression. Since this is mediated by mTOR signaling, the addition of metformin significantly attenuates the induction of LOX-1 by AGEs. This is in keeping with recent evidence suggesting that metformin has a beneficial effect on the vasculature. The United Kingdom Prospective Diabetes Study demonstrated that in obese type 2 diabetic patients, metformin reduced the risk of myocardial infarction more than sulphonyl- ureas or insulin. The vasoprotective role of metformin is largely independent of its hypoglycemic action and has been partly ascribed to the beneficial effect of metformin on body weight and plasma lipids. In addition, it has been reported that metformin has favorable pleiotropic effects on the cardiovascular system ${ }^{40,41)}$.

In conclusion, the receptor RAGE, PI3K and mTORC2/RICTOR are involved in the regulation of AGE-induced LOX-1 expression in endothelial cells (Fig.9). AGEs bind to their specific receptor RAGE, activate the PI3K/PDK1/mTORC2 signaling pathway and thereby induce LOX-1 expression. The induction of LOX-1 by AGEs may contribute to the increased atherosclerotic risk in diabetes and this process can be inhibited by metformin in vitro.

\section{Conflict of Interest}

None.

\section{References}

1) Sawamura T, Kume N, Aoyama T, Moriwake H, Hoshiawa H, Aiba Y, Tanaka T, Miwa S, Katsura Y, Kita T, Masaki T: An endothelial receptor for oxidized low density lipoprotein. Nature, 1997; 386: 73-77

2) Yoshida H, Kondratenko N, Green S, Steinberg D, Quehenberger O: Identification of the lectin-like receptor for oxidized low-density lipoprotein in human macrophages and its potential role as a scavenger receptor. Biohem J, 1998; 334(Pt1): 9-13

3) Sakata N, Uesugi N, Takebayashi S, Nagai R, Jono T, Horiuchi S, Takeya M, Itabe H, Takano T, Myint T, Taniguchi N: Glycoxidation and lipid peroxidation of lowdensity lipoprotein can synergistically enhance atherogenesis. Cardiovasc Res, 2001; 49: 466-475

4) Imanaga Y, Sakata N, Takebayashi S, Matsunaga A, Sasaki J, Arakawa K, Nagai R, Horiuchi S, Itabe Hm Takano T: In vivo and in vitro evidence for the glycosylation of lowdensity lipoprotein in human atherosclerotic plaques. Atherosclerosis, 2000; 150: 343-355

5) Mehta JL, Chen J, Yu F, Li DY: Aspirin inhibits ox-LDLmediated LOX-1 expression and metallopoteinase- 1 in human coronary endothelial cells. Cardiovasc Res, 2004; 64: 243-249

6) Li D, Singh RM, Liu L, Chen H, Singh BM, Kazzaz N, Mehta JL: Oxidized-LDL through LOX-1 increases the expression aniotensin converting enzyme in human coronary artery endothelial cells. Cardiovasc Res, 2003; 57: 238-243

7) Li D, Liu L, Chen H, Sawamura T, Mehta JL: LOX-1, an oxidized LDL endothelial receptor, induces CD40/ CD40L signaling in human coronary artery endothelial cells. Arterioscler Thromb Vasc Biol, 2003; 23: 816-821

8) Li D, Sldeen T, Romeo F, Mehta JL: Oxidized LDL upregulates angiotensin II type 1 receptor expression in cultured human coronary artery endothelial cells: the 
potential role of transcription factor NF-kappa B. Circulation, 2000; 102: 1970-1976

9) Li D, Chen H, Romeo F, Sawamura T, Saldeen T, Mehta JL: Statins modulate oxidized low-density lipoproteinmediated adhesion molecule expression in human coronary artery endothelial cells: role of LOX-1. J Pharmacol Exp Ther, 2002; 302: 601-605

10) Li D, Liu L, Chen H, Sawamura T, Ranganathan S, Mehta JL: LOX-1 mediates oxidized low-density lipoprotein-induced expression of matrix metalloproteinases in human coronary artery endothelial cells. Circulation, 2003; 107: 612-617

11) Mehta JL, Li DY, Chen HJ, Joseph J, Romeo F: Inhibition of LOX-1 by statins may relate to upregulation of eNOS. Biochem Biophys Res Commun, 2001; 289: 857861

12) Kataoka H, Kume N, Miyamoto S, Minami M, Morimoto M, Hayashida K, Hashimoto N, Kita T: Oxidized LDL modulates $\mathrm{Bax} / \mathrm{Bcl}-2$ through the lectinlike Ox-LDL receptor-1 in vascular smooth muscle cells. Arterioscler Thromb Vasc Biol, 2001; 21: 955-960

13) Li L, Sawamura T, Renier G: Glucose enhances human macrophage LOX-1 expression: role for LOX-1 in glucose-induced macrophage foam cell formation. Circ Res, 2004; 94: 892-901

14) Li D, Mehta JL: Upregulation of endothelial receptor for oxidized LDL (LOX-1) by oxidized LDL and implications in apoptosis of human coronary artery endothelial cells: evidence from use of antisense LOX-1 mRNA and chemical inhibitors. Arterioscler Thromb Vasc Biol, 2000; 20: 1116-1122

15) Aoyama T, Fujiwara H, Masaki T, Sawamura T: Induction of lectin-like oxidized LDL receptor by oxidized LDL and lysophosphatidylcholine in cultured endothelial cells. J Mol Cell Cardiol, 1999; 31: 2101-2114

16) Murase T, Kume N, Korenaga R, Ando J, Sawamura T, Masaki T, Kita T: Fluid shear stress transcriptionally induces lectin-like oxidized LDL rceptor-1 in vascular endothelial cells. Circ Res, 1998; 83: 328-333

17) Chen M, Nagase M, Fujita T, Narumiya S, Masaki T, Sawamura T: Diabetes enhances lectin-like oxidized LDL receptor-1 (LOX-1) expression in the vascular endothelium: possible role of LOX-1 ligand and AGE. Biochem Biophys Res Commun, 2001; 287: 962-968

18) Li L, Roumeliotis N, Sawamura T, Renier G: C-reactive protein enhances LOX-1 expression in human aortic endothelial cells: relevance of LOX-1 to C-reactive protein-induced endothelial dysfunction. Circ Res, 2004; 95: 877-883

19) Hofnagel O, Luechtenborg B, Stolle K, Lorkowski S, Eschert H, Plenz G, Robenek H: Proinflammatory cytokines regulate LOX-1 expression in vascular smooth muscle cells. Arterioscler Thromb Vasc Biol, 2004; 24: 17891795

20) Li DY, Zhang YC, Philips MI, Sawamura T, Mehta JL: Upregulation of endothelial receptor for oxidized lowdensity lipoprotein (LOX-1) in cultured human coronary artery endothelial cells by angiotensin II type 1 receptor activation. Circ Res, 1999; 84: 1043-1049

21) Tan KC, Shiu SW, Wong Y, Leng L, Bucala R: Soluble lectin-like oxidized low density lipoprotein receptor- 1 in type 2 diabetes mellitus. J Lipid Res, 2008; 49: 14381444

22) Iwashima Y, Eto M, Hata A, Kaku K, Horiuchi F, Ushikubi F, Sano H: Advanced glycation end productsinduced gene expression of scavenger receptors in cultured human monocyte-derived macrophages. Biophys Res Commun, 2000; 277: 368-380

23) Xie Y, Wang YL, Yu L, Hu Q, Ji L, Zhang Y, Liao QP: Metformin promotes progesterone receptor expression via inhibition of mammalian target of rapamycin (mTOR) in endometrial cancer cells. J Steroid Biochem Mol Biol, 2011; 126: 113-120

24) Zakikhani M, Dowling R, Fantus IG, Sonenberg N, Pollak M: Metformin is an AMP kinase-dependent growth inhibitor for breast cancer cells. Cancer Res, 2006; 66: 10269-10273

25) Makita Z, Vlassara H, Cerami A, Bucala R: Immunochemical detection of advanced glycosylation end products in vivo. J Biol Chem, 1992; 267: 5133-5138

26) Tan KC, Ai VH, Chow WS, Chau MT, Leong L, Lam KS: Influence of low-density lipoprotein (LDL) subfraction profile and LDL oxidation on endothelium-dependent and independent vasodilation in patients with type 2 diabetes. J Clin Endocrinol Metab, 1999; 84: 3212-3216

27) Sakata N, Uesugi N, Takebayashi S, Nagai R, Jono T, Horiuchi S, Takeya M, Itabe H, Takano T, Myint T, Taniguchi N: Glycoxidation and lipid peroxidation of lowdensity lipoprotein can synergistically enhance atherogenesis. Cardiovasc Res, 2001; 49: 466-475

28) Yan SF, Ramasamy R, Schmidt AM: The receptor for advanced glycation endproducts (RAGE) and cardiovascular disease. Expert Rev Mol Med, 2009; 11: e9. Review

29) Bucala R, Makita Z, Vega G, Grundy S, Koschinsky T, Cerami A, Vlassara H: Modification of low density lipoprotein by advanced glycation end products contributes to the dyslipidemia of diabetes and renal insufficiency. Proc Natl Acad Sci, 1994; 91: 9441-9445

30) Velarde V, Jenkins AJ, Christopher J, Lyons TJ, Jaffa AA: Activation of MAPK by modified low-density lipoproteins in vascular smooth muscle cells. J Appl Physiol, 2001; 91: 1412-1420

31) Glazer HP, Osipov RM, Clements RT, Sellke FW, Bianchi $\mathrm{C}$ : Hypercholesterolemia is associated with hyperactive cardiac mTORC1 and mTORC2 signaling. Cell Cycle, 2009; 8: 1738-1746

32) Mueller MA, Beutner F, Teupser D, Ceglarek U, Thiery J: Prevention of atherosclerosis by the mTOR inhibitor everolimus in LDLR-/- mice despite severe hypercholesterolemia. Atherosclerosis, 2008; 198: 39-48

33) Sarbassov DD, Ali SM, Kim DH, Guertin DA, Latek RR, Erdjument-Bromage H, Tempst P, Sabatini DM: Rictor, a novel binding partner of mTOR defines a rapamycininsensitive and raptor-independent pathway that regulates the cytoskeleton. Curr Biol, 2000; 14: 1296-1302

34) Jacinto E, Loewith R, Schmidt A, Lin S, Ruegg MA, Hall A, Hall MN: Mammalian TOR complex 2 controls the actin cytoskeleton and is rapamycin insensitive. Nat Cell Biol, 2004; 6: 1122-1128

35) Sarbassov DD, Guertin DA, Ali SM, Sabatini DM: Phos- 
phorylation and regulation of $\mathrm{Akt} / \mathrm{PKB}$ by the rictormTOR complex. Science, 2005; 307: 1098-1101

36) Sarbassov DD, Ali SM, Sengupta S, Sheen JH, Hsu PP, Bagley AF, Markhard AL, Sabatini DM: Prolonged rapamycin treatment inhibits mTORC2 assembly and Akt/ PKB. Mol Cell, 2006; 22: 159-168

37) Lazorchak AS, Liu D, Facchinetti V, Di Lorenzo A, Sessa WC, Schatz DG, Su B: Sin1-mTORC2 suppresses rag and il7r gene expression through Akt2 in B cells. Mol Cell, 2010; 39: 433-443

38) Mukai E, Kume N, Hayashida K, Minami M, Yamada $Y$, Seino Y, Kita T: Heparin-binding EGF-like growth factor induces expression of lectin-like oxidized LDL receptor-1 in vascular smooth muscle cells. Atherosclerosis, 2004;
176: $289-296$

39) Dormond O, Contreras GA, Meijer E, Datta D, Flynn E, Pal S, Briscoe DM: CD40-induced signaling in human endothelial cells results in mTORC2- and Akt-dependent expression of vascular endothelial growth factor in vitro and in vivo. J Immunol, 2008; 181: 8088-8095

40) Grant PJ: Beneficial effects of metformin on haemostasis and vascular function in man. Diabetes Metab, 2003; 29(4Pt2): 64S44-52

41) Anfossi G, Russo I, Bonomo K, Trovati M: The cardiovascular effects of metformin: further reasons to consider an old drug as a cornerstone in the therapy of type 2 diabetes mellitus. Curr Vasc Pharmacol, 2010; 8: 327-337 